\title{
Predicting Precipitation Kinetics During the Annealing of Additive Manufactured Inconel 625 Components
}

\author{
Magnus J. Anderson $^{1}$ (D) . Jonathan Benson ${ }^{1} \cdot$ Jeffery W. Brooks ${ }^{1} \cdot$ Benjamin Saunders $^{2} \cdot$ Hector C. Basoalto $^{1}$
}

Received: 5 December 2018 / Accepted: 22 March 2019 / Published online: 22 April 2019

(C) The Author(s) 2019

\begin{abstract}
The prediction of solidification microstructures associated with additive manufacture of metallic components is fundamental in the identification scanning strategies, process parameters and subsequent heat treatments for optimised component properties. Interactions between the powder particles and the laser heat source result in complex thermal fields in and around the metal melt pool, which will influence the spatial distribution of chemical species as well as solid-state precipitation reactions. This paper demonstrates that a multi-component, multi-phase precipitation model can successfully predict the observed precipitation kinetics in Inconel 625, capturing the anomalous precipitation behaviour exhibited in additively manufactured components. A computer coupling of phase diagrams and thermochemistry (CALPHAD)-based approach captures the impact of dendritic segregation of alloying elements upon precipitation behaviour. The model was successful in capturing the precipitation kinetics during annealing considering the $\mathrm{Nb}$-rich and $\mathrm{Nb}$-depleted regions that are formed during additive manufacturing.
\end{abstract}

Keywords Nickel-based superalloy $\cdot$ Inconel $625 \cdot$ Precipitation $\cdot$ Mean-field theory $\cdot$ Additive manufacture

\section{Introduction}

The manufacture of nickel-based superalloy components through powder-bed selective laser melting (SLM) is challenging. The interaction between the laser heat source and the randomly dispersed powder particles results in complex thermal histories characterising the process. Associated with the rapid solid-liquid-vapour transitions is the development of mechanical fields connected to the development of residual stresses. Mass transport of chemical species in the melt pool will be influenced by the solidification front during dendritic growth and promote chemical segregation [1]. Variations in chemical compositions between the interdendritic zones and the truck of dendrites will lead to heterogeneous distributions of precipitates after the application

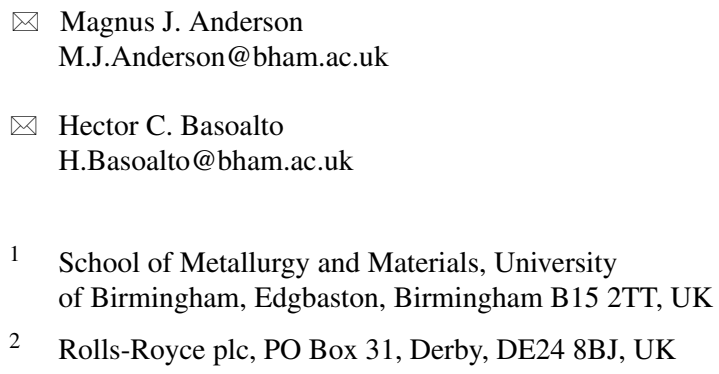

1 School of Metallurgy and Materials, University of Birmingham, Edgbaston, Birmingham B15 2TT, UK

2 Rolls-Royce plc, PO Box 31, Derby, DE24 8BJ, UK

of subsequent heat treatments. This has proven to be a major issue in the additive manufacture (AM) of $\gamma^{\prime}$ strengthened superalloys where uncontrolled precipitation of the intermetallic phase will lead to reduced ductility. Precipitation during AM will influence the mechanical response and consequently the development of component distortions and residual stresses. The ability to predict location specific microstructure variations is important in both understanding and predicting component properties following post-build heat treatments and in-service response. In addition, precipitation models can also be used to guide and optimise heat treatments and predict the evolution of Zener pinning precipitates during grain growth.

The chemical composition of the powder Inconel 625 of interest is given in Table 1 [2], showing a high amount of Niobium and low concentrations of $\gamma^{\prime}$ forming elements. The alloy is typically thought as being solid solution strengthened; however, $\gamma^{\prime \prime}$ and $\delta$ precipitates form during long exposures at high temperature [5]. Both the $\gamma^{\prime \prime}$ and $\delta$ phases are intermetallic and are formed by the ordering of niobium to create either a body centred tetragonal $\mathrm{D}_{22}$ structure or a orthorhombic D0 $a$ crystal structure, respectively. The $\gamma^{\prime \prime}$ and $\delta$ phases compete for niobium, with $\delta$ being the more thermodynamically stable phase. $\delta$ is observed to precipitate upon grain boundaries whilst $\gamma^{\prime \prime}$ precipitates heterogeneously 
Table 1 The composition of the Inconel 625 powder (wt\%) [2]

\begin{tabular}{lllllllllllll}
\hline $\mathrm{Ni}$ & $\mathrm{Cr}$ & $\mathrm{Mo}$ & $\mathrm{Nb}$ & $\mathrm{Fe}$ & $\mathrm{Ti}$ & $\mathrm{Al}$ & $\mathrm{Co}$ & $\mathrm{Mn}$ & $\mathrm{C}$ & $\mathrm{Si}$ & $\mathrm{P}$ & $\mathrm{S}$ \\
\hline bal & 20.7 & 8.83 & 3.75 & 0.72 & 0.35 & 0.28 & 0.18 & 0.03 & 0.01 & 0.13 & $<0.01$ & 0.002 \\
\hline
\end{tabular}

within grains, with preference for nucleating upon dislocations. In conventionally processed Inconel 625, Sauve et al. [3] observed $\gamma^{\prime \prime}$ to form with an initial spherical morphology and grow into lens shaped precipitates during heat treatment. The $\delta$ precipitates were observed to have plate-like morphology.

Although Inconel 625 is known to be a weldable alloy, the AM of the alloy poses a problem. The thermal loading and cooling rates for precipitation varies considerable considering different AM methods, such as electron beam melting (EBM), selective laser melting (SLM), or laserdirect metal deposition (L-DMD) [2]. For example, Lass et al. [2] did not observe any intermetallic precipitates in SLM builds, whilst Amato et al. [4] observed fine nanoscale $\gamma^{\prime \prime}$ precipitates. This suggests that the precipitation kinetics is sensitive to the process parameters for SLM powder bed processing of Inconel 625. Amato et al. [4] observed $\gamma^{\prime \prime}$ particles in both SLM and EBM builds, which formed columnar arrays of precipitates on low angle grain boundaries. The spacing of the columns, the precipitate size, and precipitate concentration varied for the two builds with larger plate-like precipitates in the EBM specimen. It is known that significant dendritic segregation of niobium forms during SLM, and is associated with anomalous precipitation kinetics during post-heat treatments, accelerating the precipitation of $\delta$ so that it is fully precipitated after $1 \mathrm{~h}$ at $870{ }^{\circ} \mathrm{C}$ [2]. For comparison, in the as-rolled condition, Sauve et al. [3] observed the onset of $\delta$ formation after $3-4 \mathrm{~h}$ at this temperature.

This paper presents the mathematical model and its implementation that can capture this behaviour. This involves using multiple techniques to calculate the thermo-mechanical loading to allow for the prediction of precipitation during SLM. The mean-field model is presented in [6], and describes the application of mean-field modelling of the intermetallic precipitates in Inconel 718, which is similar to Inconel 625 with the addition of $\gamma^{\prime}$ precipitates which form due to the larger $\mathrm{Al}$ and $\mathrm{Ti}$ content in the alloy. The model presented in this work includes refinements to the description of heterogeneous nucleation and modifications to capture the sluggish nucleation kinetics of $\gamma^{\prime \prime}$ and $\delta$ in Inconel 625 .

The paper is structured as follows. Section "Model Formulation" presents the modelling framework, outlining the mean-field model. The next section outlines the model implementation, detailing the numerical methods and input parameters. The results are then presented showing an example of the finite element analysis (FEA) predictions, and the predicted evolution of precipitates during SLM and annealing. This is followed by a discussion section, identifying strengths and weaknesses of the current model formulation. A conclusion then summarises the findings of the paper.

\section{Model Formulation}

\section{Modelling Framework}

The proposed modelling framework for the simulation of precipitation kinetics during the annealing of a SLM component is now presented. The approach involves simulation of the metal melt pool through a volume-offluid (VoF) formulation [7, 8], from which the predicted melt pool geometry and the thermal loading are used to calibrate a finite element (FE) model of the heat source model when simulating the AM of a build [6]. The process-induced thermo-mechanical fields predicted by the $\mathrm{FE}$ analysis provide the driving forces required for solid-state precipitation reactions. Coupling of these fields to a CALPHAD thermodynamic approach, the influence of composition on phase transformations can be modelled. Statistics of the precipitation distributions are tracked through a composition dependent mean-field model of the precipitation size distribution, which accounts for nucleation, growth, coarsening and dissolution of precipitate phases. Figure 1 gives an overview of how these methods have been integrated.

\section{Component-Scale Modelling}

The simulation of an SLM build through a FE implementation is challenging due to the different length scales characterising the deposition process, heat source and size of the component. Observations of melt pool geometries suggest that large spatial gradients of temperature are generated within a depth of $\approx 80 \mu \mathrm{m}$. To ensure the accurate prediction of the formation of thermal strains incurred by such thermal loading, ideally the mesh size needs to be less than $20 \mu \mathrm{m}$. As a result, the computational cost of simulating geometries greater than $1 \mathrm{~mm}^{3}$ with accuracy becomes prohibitive. To make simulations in a reasonable amount of time, methods for homogenising the deposition of material are needed. One approach is to calculate an eigenstrain that is characteristic of the specific SLM process and material, and then apply this eigenstrain to elements as they are activated, capturing the distortion of the geometry during the 


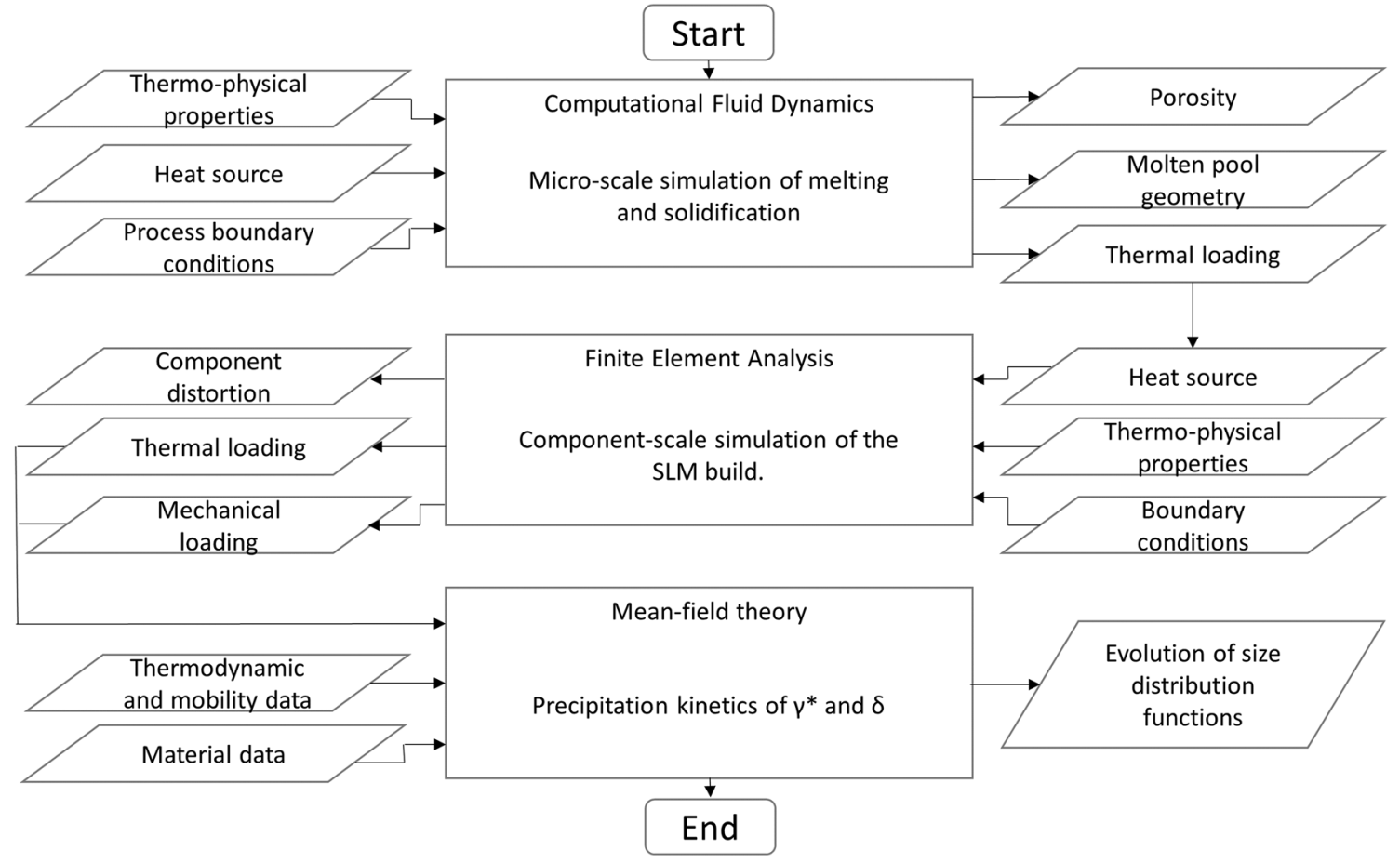

Fig. 1 The modelling framework applied to predict precipitation kinetics during annealing of a SLM component

build. Alternatively, the thermal loading can be scaled to describe a larger deposition of material, simulating the SLM process and capturing the heat dissipate within the geometry of interest. The latter approach was adopted in this work as it allows for the calculation of location specific thermal loading in the component geometry, and is described in [6].

The commercial finite element package Abaqus was used to simulate the SLM process based on a decoupled thermal and mechanical analysis. The thermo-physical properties for Inconel 625 were obtained from Capriccioli and Frosi [9]. A Python script was written to automate the preprocessing operations. User subroutines were implemented, as described by Anderson et al. [6]. This includes the "property switching function", a description of the heat source, and a subroutine for updating boundary conditions. The thermal load in the FE model was calibrated so that the calculated peak temperatures aligned with predictions made using VoF approach [7, 8].

\section{Mean-Field Precipitation Modelling}

As already mentioned a mean-field description is adopted to describe the evolution of the $\gamma^{\prime \prime}$ and $\delta$ precipitates, assuming weak interaction between particles. The precipitate morphology of $\gamma^{\prime \prime}$ and $\delta$ are simplified to be described by cylinders, and are characterised by the particle radius frequency density function, $\mathcal{F}(R, t)$, where $R$ refers to the radius of a sphere of equivalent volume to the cylindrical particles of interest. Let $\mathcal{F}(R, t) d R$ describe the number of particles with a radius within the closed interval of $R$ and $R+d R$ within a representative unit volume. The mean particle radius $\langle R\rangle$, the particle concentration $N_{v}$ and the volume fraction $\phi$ are obtained from moments of the $\mathcal{F}(R, t)$ function. The $D$ th moment of $\mathcal{F}(R, t)$ is given by the following:

$M^{(D)}=\int_{0}^{\infty} \mathcal{F}(R, t) R^{D} \mathrm{~d} R$

The particle concentration $N_{v}$ is given by $M^{(0)}$, the mean particle radius $\langle R\rangle=M^{(1)} / M^{(0)}$, and the volume fraction $\phi=(4 \pi / 3) M^{(3)}$. The evolution of the precipitate dispersion is governed by the following advection equation:

$\frac{\partial \mathcal{F}(R, t)}{\partial t}+\frac{\partial(\mathcal{F}(R, t) \dot{R}(R, t))}{\partial R}=\mathcal{F}^{+}(R, t)-\mathcal{F}^{-}(R, t)$

where $\mathcal{F}^{+}(R, t)$ and $\mathcal{F}^{-}(R, t)$ are source and sink terms, describing mechanisms that affect the particle concentration, $N_{v}$. In this model, they refer to nucleation and dissolution, respectively. However, they can also include precipitate coalescence phenomena [10]. The particle growth rate has the following generic form:

$\dot{R}(R, t)=\frac{A(t)}{R}\left(\frac{1}{R_{c}(t)}-\frac{1}{R}\right) z(R, t)$

where the term $A(t)$ includes the diffusivity of the alloying elements, $R_{c}(t)$ is the critical particle radius and $z(R, t)$ 
is the competitive growth correction factor. Marqusee and Ross [14] derived the following expression for the $z(R, t)$ factor as follows:

$z(R, t)=1+R \sqrt{4 \pi M^{(1)}}$

in the present work, it will be assumed that $\delta$ and $\gamma^{\prime \prime}$ precipitates do not directly compete for solute as $\delta$ nucleates at grain boundaries and $\gamma^{\prime \prime}$ nucleates within the grain interior, so that each phase has a separate $z(R, t)$ factor.

To describe the kinetics of $\gamma^{\prime \prime}$ and $\delta$, the multi-component particle growth model proposed by Svoboda-Fischer-FratzlKozeschnik (SFFK) [11] has been implemented. This framework was originally developed for spherical particles. To account for other precipitate morphologies, the shape factors derived by Kozeschnik et al. [12] and Svoboda et al. [13] have been used to describe the $\gamma^{\prime \prime}$ and $\delta$ phases as cylinders opposed to spheres, with an aspect ratio given by $h=H / D$, where $H$ is the height of the cylinder and $D$ is the diameter. Combining the SFFK model and the shape parameters of Kozeschnik et al.'s [12], the following expressions for the parameters for the particle growth rate given by Eq. 3 are obtained as follows:

$$
\begin{aligned}
A(t) & =\frac{S_{k}}{O_{k}} \frac{2 \gamma \theta}{R_{g} T} \\
R_{c}(t) & =\frac{2 \gamma S_{k}}{\Delta G_{c}} \\
\theta & =\left[\sum_{i=1}^{n} \frac{\left(c_{k i}-c_{0 i}\right)^{2}}{c_{0 i} D_{0 i}}\right]^{-1} \\
\Delta G_{c} & =-U-\sum_{i=1}^{n} c_{k i}\left(\mu_{k i}-\mu_{0 i}\right) \\
U & =\frac{\varepsilon^{2} E}{1-v}
\end{aligned}
$$

where $\gamma$ is the interfacial energy, $R_{g}$ is the gas constant, and $T$ is the absolute temperature. The terms $c_{k i}$ and $c_{0 i}$ refer to the chemical concentration of the $i$ th alloying element in the particle and matrix phase, respectively. Similarly, $\mu_{k i}$ and $\mu_{0 i}$ refer to chemical potentials in the matrix and particle for the $i$ th alloying element, and $D_{0 i}$ is the diffusivity of the $i$ th alloying element. $U$ is the misfit strain energy caused by differences in the lattice parameters between the precipitate and matrix phases, where the misfit strain is given by $\varepsilon$. $E$ and $v$ refer to the Young's modulus and Poisson's ratio. $S_{k}$ and $O_{k}$ are shape parameters and are functions of the precipitate aspect ratio, $h$.

Nucleation is treated as outlined by Anderson et al. [6] as follows:

$\mathcal{F}^{+}(R, t)=Z \beta^{*}(t) N_{c}(R, t) \exp \left(\frac{-\Delta G^{*}}{k_{b} T}\right) P_{\text {inc }}$

where the Zeldovitch parameter $Z, k_{b}$ is the Boltzmann constant, and the form of the nuclei radius distribution function $N_{c}(R, t)$ are obtained from Jou et al. [15] as follows:

$N_{c}(R, t)=\frac{N_{0}}{\Delta R \sqrt{2 \pi}} \exp \left(-\frac{1}{2}\left(\frac{R-R_{c}(t)}{\Delta R}\right)^{2}\right)$

$\Delta R$ is approximated by the following:

$\Delta R=\left(\frac{3 \Omega}{2(\pi)^{3 / 2}} \frac{1}{Z}\right)^{\frac{1}{3}}$

where $\Omega$ is the atomic volume. $N_{0}$ is the concentration of nuclei, and is estimated from the supersaturation of precipitate forming species as follows:

$N_{0}=\eta \frac{3\left(\phi_{\mathrm{eq}}-\phi(t)\right)}{4 \pi R_{c}^{3}}$

where $\phi_{\text {eq }}$ is the equilibrium volume fraction of precipitates. For the $\delta$ and $\gamma^{\prime \prime}$ phases, the volume fraction $\phi(t)$ that enters Eq. 9 is the total volume fraction of the combined $\delta$ and $\gamma^{\prime \prime}$ precipitates. The term $\eta$ refers to the fraction of active nucleation sites. For homogeneous nucleation, $\eta$ is unity. To approximate $\eta$ for heterogeneous nucleation, $\eta$ is given by the ratio of the available nucleation sites divided by the total number of nucleation sites within the volume of interest.

For heterogeneous nucleation upon dislocations within grains, $\eta$ will be assumed to be given by the fraction of lattice sites occupied by dislocations. Let the dislocation density be defined as the total length of dislocations found within a volume, $V$ so that $\rho=L / V$. The number of lattice sites along dislocations is given by $L / b$, where $b$ is the Burgers vector. The total number of lattice sites within the volume can be determined by $V b^{-3} . \eta$ is then given by the following:

$\eta=b^{2} \rho$

To complete the last expression, an estimate of the dislocation density is needed. It will be assumed that the thermo-mechanical fields induced by the deposition process will result in localised plastic distortions. Following Kocks et al. [16], the development of the dislocation density can be modelled through a continuity condition considering dislocation generation and annihilation mechanisms. Assuming that the generation rate scales with the dislocation density and dipole formation is the dominant annihilation mechanism, it can be shown that the dislocation evolution has the form as follows:

$\dot{\rho}=\mathcal{C}_{\rho} \sqrt{\rho}\left(1-\sqrt{\frac{\rho}{\rho_{s}}}\right)\left|\dot{\varepsilon}_{p}\right|$

where $\dot{\epsilon}_{p}$ is the plastic rate, $C_{\rho}$ is a rate constant, and $\rho_{s}$ is the steady-state dislocation density. The steady-state dislocation density $\rho_{s}$ is given by the following [17]:

$\rho_{s}=\left(\frac{\pi(1-v) \sigma}{M b G}\right)^{2}$ 
where $M$ is the Taylor factor, $G$ is the shear modulus, $v$ is Poisson's ratio, and $\sigma$ is the effective stress.

It is proposed that grain boundary dislocations act as nucleation sites for grain boundary precipitates. A lower bound estimate for the grain boundary dislocation density can be made by determining the dislocations needed to accommodate differences in lattice orientation between neighbouring grains. The dislocation density at the grain boundaries is likely to be higher, with additional dislocations formed during plastic deformation, and geometrically necessary dislocations that accommodate plastic strain. Carbides with incoherent lattice alignment with the neighbouring matrix would also provide additional dislocations where precipitates could nucleate. Let $\rho_{g b}$ refer to the mean dislocation density at the grain boundaries. The number of lattice sites that are found on grain boundary dislocations on a grain of diameter $\langle d\rangle$ is given by $\rho_{g b} \pi\langle d\rangle^{2}$. The fraction of nucleation sites considering grain boundaries is then given by the following:

$\eta=\frac{\rho_{g b} \pi\langle d\rangle^{2}}{\Omega}$

Figure 2 illustrates the fraction of active nucleation sites considering heterogenous nucleation upon dislocations and upon grain boundaries, respectively.

The term $\beta^{*}$ is the atomic attachment rate, which uses the multi-component approximation derived by Svoboda et al. [11]:

$\beta^{*}=\frac{4 \pi R_{c}^{2}}{a^{4} \bar{V}_{m}} \theta$

The energy barrier to nuclei formation $\Delta G^{*}$ shown in Eq. 6 is given by the following [6]:

$\Delta G^{*}=\frac{1}{\psi^{3}} \frac{16 \pi}{3} \frac{\gamma^{3}}{\left(\Delta G_{c}\right)^{2}}$ where $\psi$ is the sphericity of the nuclei, and for cylindrical precipitates with an aspect ratio of $h$ is given by the following:

$\psi=\left(h+\frac{1}{2}\right)^{-1}\left(\frac{3}{2} h\right)^{2 / 3}$

$P_{\text {inc }}$ is the nuclei incubation probability, and is given by the following:

$P_{\text {inc }}=\exp \left(\frac{-\tau}{t}\right)$

where $\tau$ is the incubation time. To determine the incubation probability during transient thermal loading, the incubation probability $P_{\text {inc }}$ is integrated numerically using the temporal derivative to Eq. 17 as follows [6]:

$\frac{d P_{\text {inc }}}{d t}=\mathcal{C}_{\tau} \frac{\tau}{t_{\mathrm{eq}}} P_{\mathrm{inc}}\left[\frac{1}{t_{\mathrm{eq}}}+\left(\frac{1}{\theta} \frac{d \theta}{d T}-\frac{2}{R_{c}} \frac{d R_{c}}{d T}+\frac{1}{\gamma} \frac{d \gamma}{d T}-1\right) \frac{d T}{d t}\right]$

where $t_{\mathrm{eq}}$ is the equivalent incubation time, $t_{\mathrm{eq}}=$ $-\tau\left[\ln \left(P_{\mathrm{inc}}\right)\right]^{-1}$. The temperature dependent material coefficient $\mathcal{C}_{\tau}$ was introduced to delay nucleation kinetics in order to better capture the sluggish precipitation behaviour exhibited by Inconel 625 .

\section{Model Implementation}

\section{Normalisation}

The nucleation, growth, coarsening, and dissolution kinetics are calculated by solving the advection equation shown in Eq. 2 for each precipitate phase. To mitigate loss of significance errors and improve numerical stability, the problem is normalised in both space and time. Let $R_{k}$ and
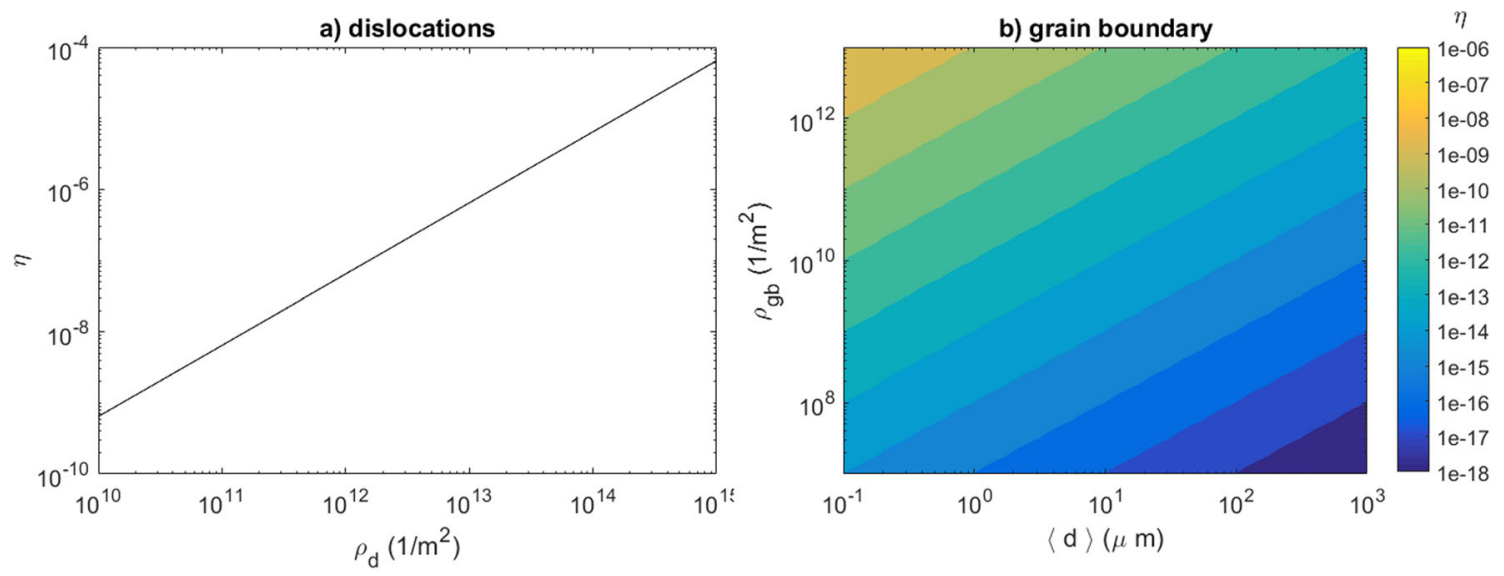

Fig. 2 The heterogeneous nucleation site fractions at $\mathbf{a}$ dislocations within grains and $\mathbf{b}$ at grain boundaries 
Table 2 The compositions used for precipitate simulations (at $\%)$

\begin{tabular}{lllllllll}
\hline Condition & $\mathrm{Al}$ & $\mathrm{Co}$ & $\mathrm{Cr}$ & $\mathrm{Fe}$ & $\mathrm{Mo}$ & $\mathrm{Nb}$ & $\mathrm{Ni}$ & $\mathrm{Ti}$ \\
\hline Bulk & 0.62 & 0.18 & 23.72 & 0.77 & 5.17 & 2.41 & bal & 0.44 \\
SLM, Nb-rich & 0.63 & 0.19 & 24.15 & 0.78 & 6.86 & 3.81 & bal & 0.44 \\
SLM, Nb-depleted & 0.62 & 0.18 & 23.73 & 0.77 & 5.81 & 1.80 & bal & 0.44 \\
\hline
\end{tabular}

$\Lambda$ refer to the spatial and temporal normalisation constants. Each precipitate phase has it's own normalisation constants, where $\Lambda=R_{k}^{3} / A$. The term $A$ is given in Equation set (3). $R_{k}$ is either the mean precipitate radius or set to a minimum value for conditions where there are no precipitates. The spatial normalisation is updated regularly, to follow the dispersion. Let $f\left(r, t^{\prime}\right) d r \equiv \mathcal{F}(R, t) R_{k}^{3} d R$, where $f\left(r, t^{\prime}\right)$ refers to the normalised particle radius distribution function. The normalised time and precipitate radius is given by $t^{\prime} \equiv$ $t / \Lambda$ and $r \equiv R / R_{k}$, respectively. The normalised continuity equation is given by the following:

$$
\frac{\partial f\left(r, t^{\prime}\right)}{\partial t^{\prime}}+\frac{\partial\left(f\left(r, t^{\prime}\right) \dot{r}\left(r, t^{\prime}\right)\right)}{\partial r}=f^{+}\left(r, t^{\prime}\right)-f^{-}\left(r, t^{\prime}\right)
$$

The normalised growth rate, $\dot{r}\left(r, t^{\prime}\right)$ is then given by the following:

$\dot{r}\left(r, t^{\prime}\right)=\frac{1}{r}\left(\frac{1}{r_{c}\left(t^{\prime}\right)}-\frac{1}{r}\right)\left(1+r \sqrt{4 \pi m^{(1)}}\right)$

where $r_{c}\left(t^{\prime}\right) \equiv R_{c} / R_{k}$ and $m^{(1)}$ refers to the first moment of the $f\left(r, t^{\prime}\right)$ function. The normalised nucleation rate is given by the following:

$$
\begin{aligned}
f^{+}\left(r, t^{\prime}\right) & =Z \beta^{\prime}\left(t^{\prime}\right) n_{c}\left(r, t^{\prime}\right) \exp \left(\frac{-\Delta G^{*}}{k_{b} T}\right) P_{\text {inc }} \\
n_{c}\left(r, t^{\prime}\right) & =\frac{n_{0}}{\sqrt{\pi \lambda}} \exp \left(-\frac{\left(r-r_{c}\right)^{2}}{\lambda}\right) \\
\beta^{\prime}\left(t^{\prime}\right) & =\frac{R_{g} T}{\gamma^{\prime}} \frac{O_{k}}{S_{k}} \frac{2 \pi r_{c^{4}}}{a^{\prime^{4}} V_{m}^{\prime}}
\end{aligned}
$$

with the following normalisation parameters as follow:

$$
N_{0} \equiv \frac{n_{0}}{R_{k}^{3}} \quad \lambda \equiv 2\left(\frac{\Delta R}{R_{k}}\right)^{2} \quad \gamma \equiv \frac{\gamma^{\prime}}{R_{k}^{2}} \quad V_{m} \equiv V_{m}^{\prime} R_{k}^{3}
$$

Equation 19 is solved using an explicit upwind finite difference scheme. The spatial discretisation used to generate the particle radius distribution functions are remeshed to follow the dispersions during the simulation. The values of $\mathcal{F}(R, t)$ which are above a certain limit are interpolated onto the new mesh using a cubic spline function, with linear interpolation applied to the remaining $\mathcal{F}(R, t)$ function.

\section{CALPHAD}

The multi-component, multi-phase mean-field model requires chemical potentials, phase chemistries, and diffusivities. The CALPHAD solver ThermoCalc [18] has been used to calculate these variables, using the thermodynamic database TTNi8, combined with the mobility database MOBNi1. A FORTRAN programme has been written which is coupled with ThermoCalc's [18] TQ FORTRAN interface. The phase diagram, phase chemistries, and equilibrium chemical potentials are calculated prior to the precipitation calculations. Look-up tables store this information, and are interpolated when calculating the evolution of the precipitate dispersions. During the precipitation calculation, the matrix composition is determined using mass balance, and
Fig. 3 a The property diagrams with the $\delta$ phase suspended. b The property diagrams with both $\gamma^{\prime \prime}$ and $\delta$
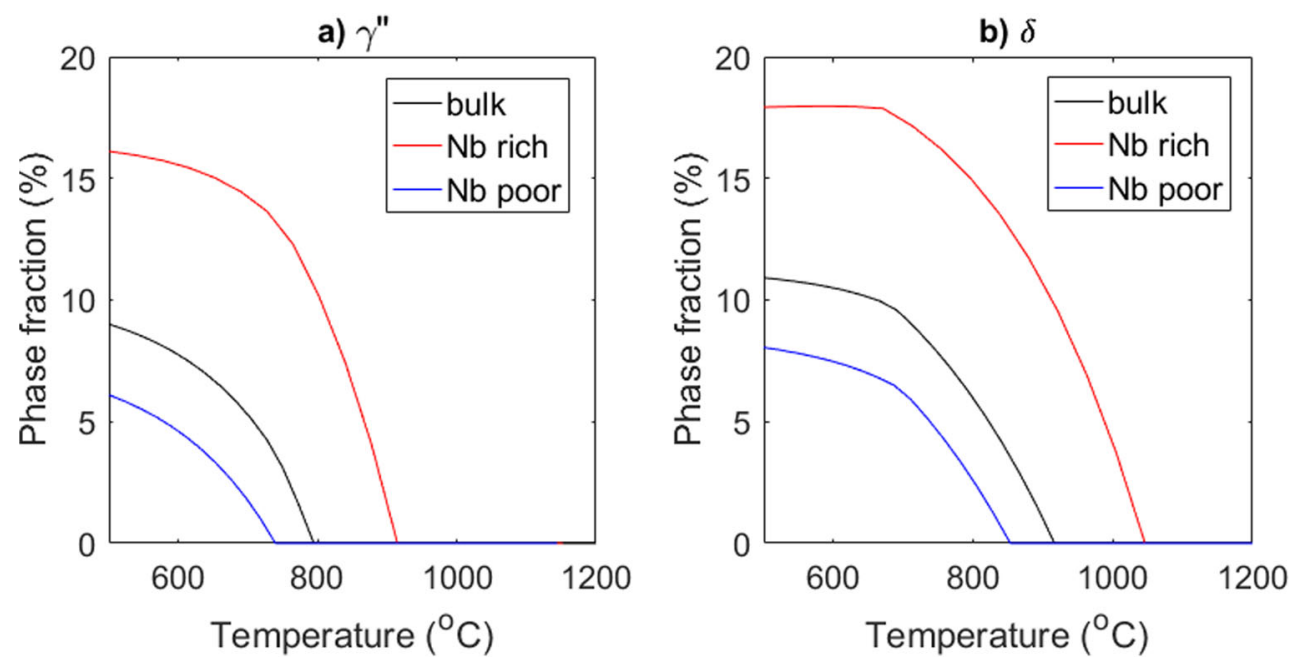
Table 3 Model parameters

\begin{tabular}{lllll}
\hline $\bar{V}_{m}$ & $\varepsilon\left[\gamma^{\prime \prime}\right]$ & $\varepsilon[\delta]$ & $\eta\left[\gamma^{\prime \prime}\right]$ & $\eta[\delta]$ \\
\hline $7.30 \mathrm{E}-06$ & $0.3 \%$ & $1 \%$ & $1 . \mathrm{E}-05$ & $1 . \mathrm{E}-15$ \\
\hline
\end{tabular}

the matrix chemical potential and diffusivities are then calculated, allowing for the determination of the precipitate growth and nucleation rates for each phase.

The simplified compositions used to model Inconel 625 in the conditions of interest are presented in Table 2. The extent of segregation in the AM condition was approximated from the energy dispersive X-ray spectrometry measurements presented by Zhang et al. [19].

\section{Calibration}

The precipitation model was calibrated using available data that describes the material in conventionally manufactured conditions. To capture the time-temperature-transition (TTT) data of Suave et al. [3], energy contributions of 500 and $-500 \mathrm{~J} / \mathrm{mol}$ were added to the $\gamma^{\prime \prime}$ and $\delta$ phases, respectively. Figure 3 presents the calculated property diagram for the $\gamma^{\prime \prime}$ and $\delta$ phases for the chemistries shown in Table 2. Figure $3 a$ and $b$ describes the calculated phase fractions of $\gamma^{\prime \prime}$ and $\delta$, respectively as a function of temperature. Three phases are considered in the thermodynamic calculations; the $\gamma, \gamma^{\prime \prime}$, and $\delta$ phases. To evaluate the metastable $\gamma^{\prime \prime}$ phase, it is necessary to suspend the $\delta$ phase from the calculation. The chemistries examined show the predicted phase fraction for the bulk composition, and how this differs when considering the Nb-rich and $\mathrm{Nb}$-depleted regions within the SLM component of interest.

The molar volume, misfit strains, and nucleation site fractions are given in Table 3. The coefficients for a polynomial temperature dependency for the Young's modulus and Poisson's ratio are given in Table 4, where the polynomial coefficients are defined by the following:

$x=\sum_{k=1}^{n} p_{k} T^{k-1}$

where $x$ is the variable of interest, the temperature, $T$, in Eq. 23 is given in degrees Celsius and $n$ refers to the
Table 5 Temperature dependency of the interfacial energy

\begin{tabular}{llllll}
\hline $\mathrm{T}$ & ${ }^{\circ} \mathrm{C}$ & 550 & 680 & 750 & 900 \\
\hline$\gamma\left[\gamma^{\prime \prime}\right]$ & $\mathrm{J} / \mathrm{m}^{2}$ & 0.025 & 0.025 & 0.025 & 0.025 \\
$\gamma[\delta]$ & $\mathrm{J} / \mathrm{m}^{2}$ & 0.025 & 0.025 & 0.03 & 0.005 \\
\hline
\end{tabular}

number of polynomial coefficients, $p_{k}$. The temperature dependency of the interfacial energy and incubation probability coefficient for the $\gamma^{\prime \prime}$ and $\delta$ phases are presented in Tables 5 and 6, respectively.

The approximation for the aspect ratio of $\gamma$ " precipitates as a function of the equivalent precipitate radius was obtained from Moore et al. [20], and is shown in Fig. 4a. The aspect ratio of $\delta$ precipitates was approximated as 0.2 . Figure $4 \mathrm{~b}$ compares the predicted coarsening kinetics of $\gamma^{\prime \prime}$ at $650{ }^{\circ} \mathrm{C}$ with data from Moore et al. [20] and Suave et al. [3].

Figure 5 compares the calibrated TTT diagram with the measurements of Suave et al. [3]. The data refers to Inconel 625 in the as-rolled (AR) and shear-span (SS) conditions, which exhibit different precipitation kinetics. These differences may be caused by differing grain size, dislocation density, segregation of alloying elements, and residual stresses considering the two material conditions. The simulations assume that the material has been quenched from $1200{ }^{\circ} \mathrm{C}$ to room temperature at $-5{ }^{\circ} \mathrm{C} / \mathrm{s}$, and heated to temperature at $5{ }^{\circ} \mathrm{C} / \mathrm{s}$.

\section{Results}

The finite element model described in [6] was applied to predict the thermo-mechanical loading during AM of the geometry illustrated in Fig. 6a. The component geometry contains several arches. The supporting material on either side of the arches are referred to as legs. Precipitation simulations have been performed for the first two legs, showing similar behaviour. Figure $6 \mathrm{~b}$ provides an example of the thermal loading predicted within the centre of the vertical cross section taken through the first leg.

The precipitation kinetics of $\gamma^{\prime \prime}$ and $\delta$ was calculated accounting for the segregation of alloying elements, shown in Table 2. The dislocation density in the AM condition was approximated by the steady-state dislocation density
Table 4 Polynomial coefficients for Young's modulus and Poisson's ratio

\begin{tabular}{lllll}
\hline Parameter & $\mathrm{p}_{1}$ & $\mathrm{p}_{2}$ & $\mathrm{p}_{3}$ & $\mathrm{p}_{4}$ \\
\hline Young's modulus (Gpa) & $2.2120 \mathrm{E}+02$ & $-3.0111 \mathrm{E}-02$ & $-6.9761 \mathrm{E}-05$ & $2.2057 \mathrm{E}-08$ \\
Poisson's constant & $3.12743 \mathrm{E}-01$ & $3.29272 \mathrm{E}-05$ & $-3.75481 \mathrm{E}-09$ & $0.00 \mathrm{E}+00$ \\
\hline
\end{tabular}


Table 6 Temperature dependency of the incubation probability coefficient

\begin{tabular}{|c|c|c|c|c|}
\hline $\mathrm{T}$ & ${ }^{\circ} \mathrm{C}$ & 650 & 800 & 930 \\
\hline $\mathcal{C}_{\tau}\left[\gamma^{\prime \prime}\right]$ & & $1.00 \mathrm{E}-03$ & $1.00 \mathrm{E}-03$ & $1.00 \mathrm{E}-03$ \\
\hline $\mathcal{C}_{\tau}[\delta]$ & & $1.00 \mathrm{E}-04$ & $1.00 \mathrm{E}-05$ & $1.00 \mathrm{E}-05$ \\
\hline
\end{tabular}

described in Eq. 12. The predicted von Mises stress and dislocation site fraction in leg 1 is shown in Fig. 7. The $\delta$ nucleation site fraction and the incubation coefficient of $\delta$ were both assumed to be $10 \times$ larger than in the conventional processed condition due to changes in grain boundary dislocation content and grain size.

No significant precipitation was predicted in the AM condition in either the $\mathrm{Nb}$-rich or $\mathrm{Nb}$-depleted regions. Neither $\gamma^{\prime \prime}$ or $\delta$ was predicted to form during annealing within the $\mathrm{Nb}$-depleted regions. The predicted size and volume fraction of the cylindrical $\gamma^{\prime \prime}$ and $\delta$ precipitates in the Nb-rich annealed condition are shown in Fig. 8. The contour map of $\gamma^{\prime \prime}$ follows the same shape as the contour of the dislocation nucleation site fraction $\eta$ shown in Fig. 7; however, the differences in statistics across the geometry is minimal. The temporal evolution of the size and volume fraction of $\gamma^{\prime \prime}$ and $\delta$ at the centre of the vertical cross section of leg1 is given in Fig. 9. The predicted TTT kinetics for the AM condition is presented in Figs. 10 and 11, for the $\mathrm{Nb}$-rich and $\mathrm{Nb}$-depleted regions respectively. The model predictions are in reasonable agreement with Stoudt et al. [21], however over-predicts kinetics at high temperatures.

\section{Discussion}

The model predictions presented in this work show reasonable agreement with the experimental data, however

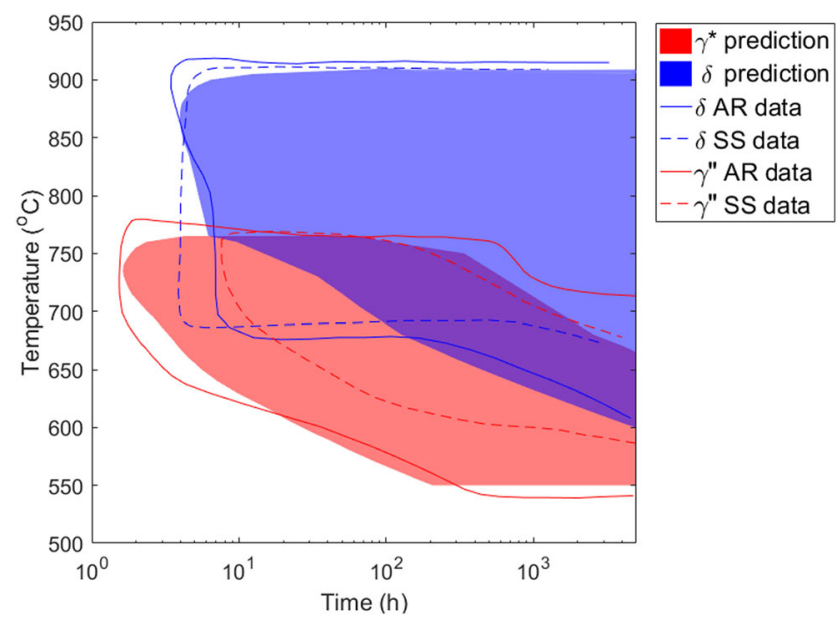

Fig. 5 The simulated TTT diagram for the bulk composition compared with the TTT measurements from Suave et al. [3] for Inconel 625 in the as-rolled (AR) and shear-span (SS) conditions

deviate at high temperature considering the TTT kinetics in the SLM condition as shown in Fig. 10. The $\delta$ precipitates are predicted to be stable at higher temperatures than Stoudt et al. [21] observed experimentally. Lindwall et al. [22] have also modelled precipitation kinetics during the heat treatment of AM Inconel 625, and have captured this behaviour. The approaches are similar in the use of FEA to predict the thermal history during AM and application of CALPHAD-based mean-field modelling of precipitation kinetics. They have demonstrated the use of the commercial software DICTRA [18] to simulate the formation of dendritic segregation of alloying elements during SLM, and have used TC-PRISMA [18] to simulate precipitation kinetics for different chemistries predicted by the DICTRA calculation.

Both the calculations presented in this paper and those by Lindwall et al.'s [22] share a similar problem in that the simulations do not account for any homogenisation
Fig. 4 a The approximated aspect ratio for $\gamma^{\prime \prime}$ as a function of equivalent radius. $\mathbf{b}$ The comparison of simulated precipitate kinetics of $\gamma *$ at $650{ }^{\circ} \mathrm{C}$ with data from Moore et al. [20] and Suave et al. [3] a)

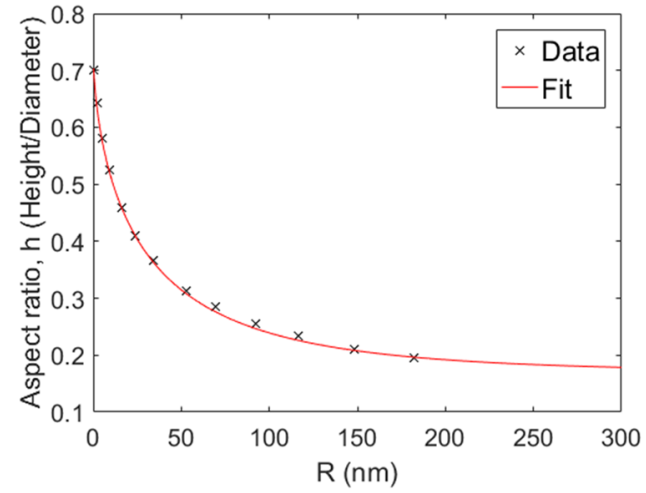

b)

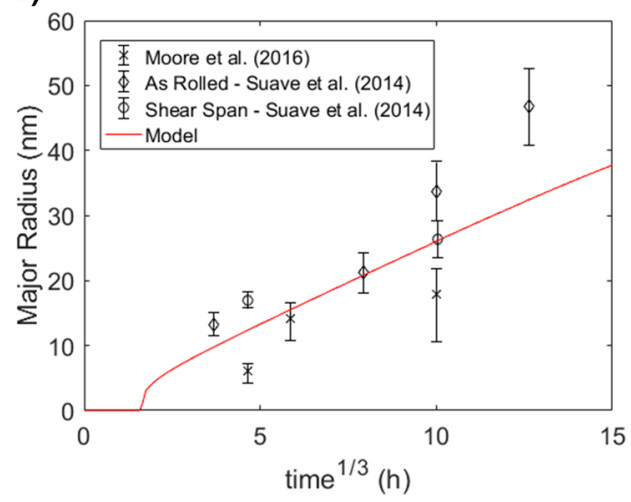


Fig. 6 a The component geometry of interest. b An example of the predicted thermal loading
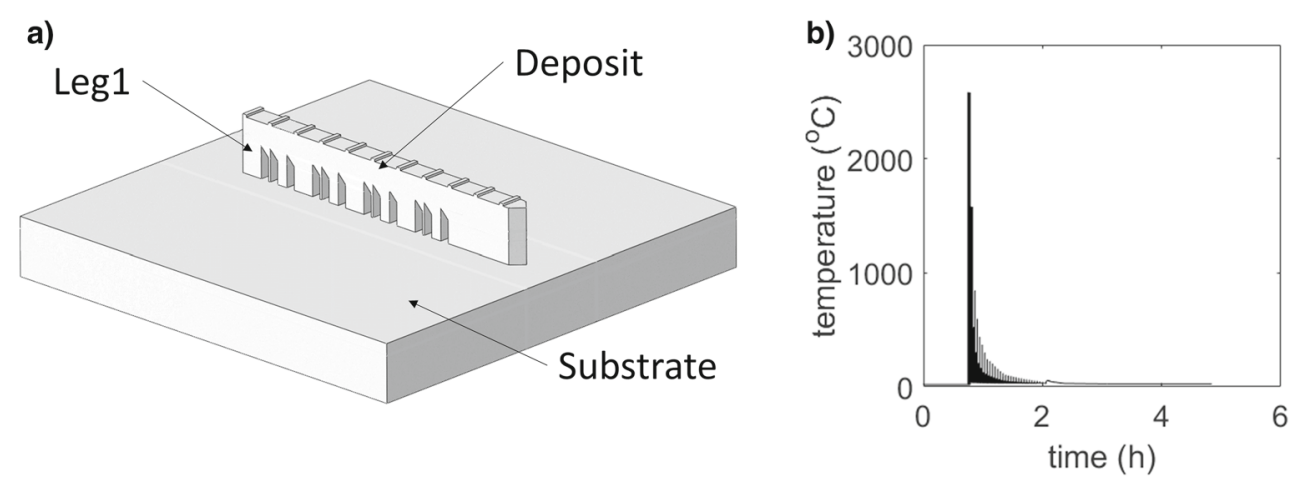

that occurs during post-build solid solution heat treatments. Lindwall et al. [22] have captured the high temperature TTT kinetics shown in Fig. 10 through calibration of the interfacial energy and nucleation site concentration during conditions where the homogenisation of the dendritic segregation of $\mathrm{Nb}$ would impact precipitation behaviour. One concern is that such a model calibration may adversely impact the predictive capability when applied to other AM conditions where the degree of segregation of alloying elements may vary.

This work aimed at identifying model parameters that can capture precipitation behaviour in both conventionally processed and SLM conditions to give greater confidence when applying the model to new conditions. It was assumed that the interfacial energy did not change significantly for different processing conditions and that the differences in precipitation behaviour are caused by the extent of the segregation of alloying elements and differences in nucleation site densities. No attempts were made to calibrate the model to capture the high temperature TTT behaviour in the AM condition as the model does not yet account for the homogenisation of the dendritic segregation which would impact the property diagram and the chemical driving force for the precipitate phase transformations.

To improve upon the accuracy of the precipitation kinetics at long ageing times and during solid solution treatments, it is necessary to couple the precipitation calculation within the multi-component diffusion model simulating dendritic segregation. This would allow for the simulation of the formation of dendritic segregation during SLM followed by the homogenisation of the diffusion fields during subsequent heat treatments.

The precipitation kinetics in Inconel 625 are slow compared to Inconel 718, which contains higher amounts of $\mathrm{Nb}$ - and $\gamma$ '-forming elements. A phenomenological parameter was introduced to delay nucleation, and better capture the precipitate behaviour. Brooks and Bridges [23] proposed that the slower kinetics of $\gamma^{\prime \prime}$ and $\delta$ in Inconel 718 is a result of the slower diffusivity of $\mathrm{Nb}$. However, the diffusivity of $\mathrm{Nb}$ obtained from the MOBNi1 and MOBNi2 mobility databases are not significantly slower than $\gamma^{\prime}$ forming species. It is possible that the $\mathrm{Nb}$ diffusivities obtained from these databases are not accurate, however attempts to artificially reduce the diffusivity of $\mathrm{Nb}$ did not
Fig. 7 a The predicted von Mises stress at leg 1. b The dislocation nucleation site fraction
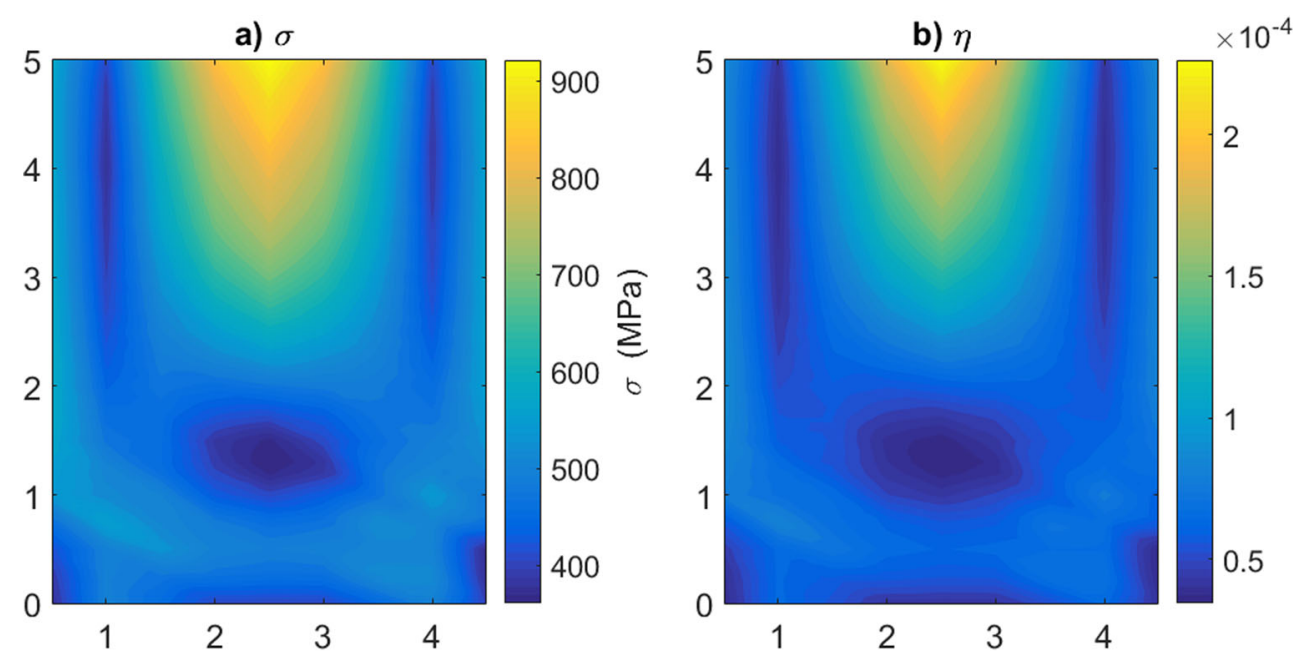
Fig. 8 Contour maps of the predicted volume fraction, mean diameter, and mean height of the $\gamma^{\prime \prime}$ and $\delta$ precipitates in the annealed condition. Panel figures a, c, and e refer to the $\gamma^{\prime \prime}$ phase and panel figures $\mathbf{b}, \mathbf{d}$, and $\mathbf{f}$ refer to the $\delta$ phase
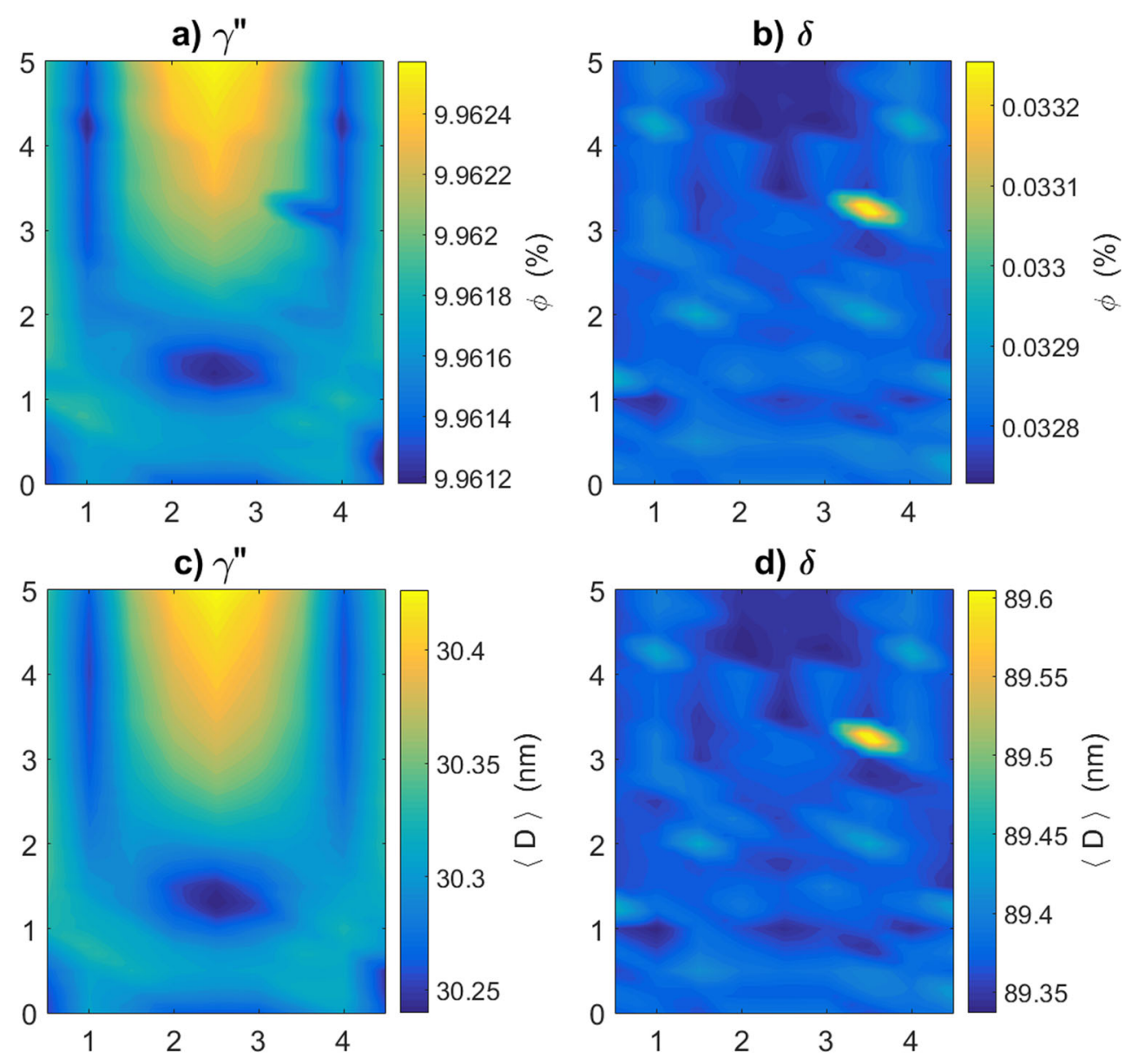

30.4
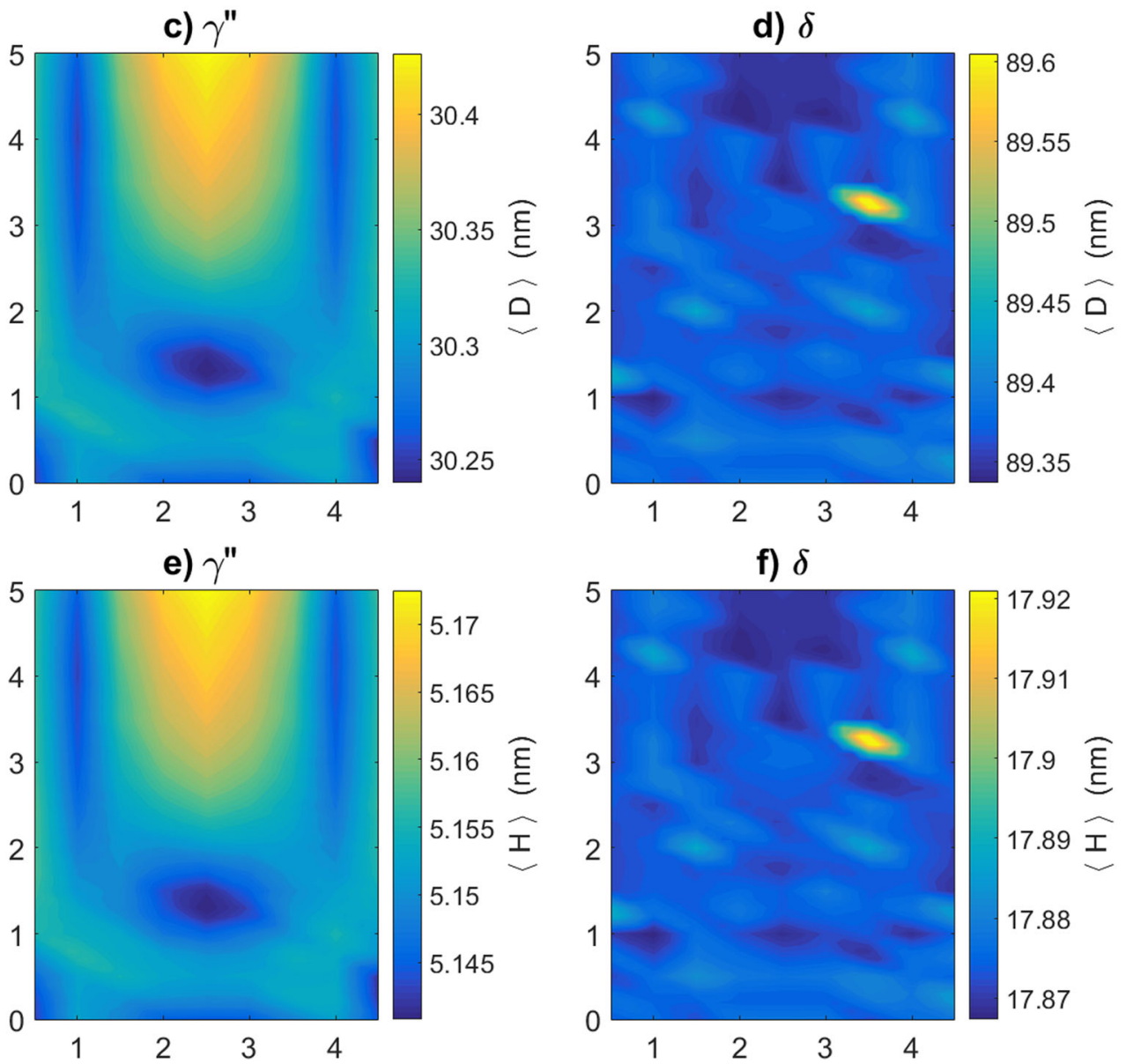

have a significant impact upon the calculated incubation time or the growth rates of $\gamma^{\prime \prime}$.

The particle growth rates of Svoboda et al. [13] and Moore et al. [20] go into greater detail regarding the description of the interfacial energy, precipitate misfit strain energy and the evolution of the aspect ratio. The evolution of the aspect ratio of the precipitates can be calculated as a function of the interfacial energy on the planar $\left(\gamma^{p}\right)$ and curved surfaces $\left(\gamma^{c}\right)$ of the cylinder and consider the misfit strain energy as a function of aspect ratio $(U(h))$. The particle growth rate of Moore et al. [20] may be expressed in the generic form given in Eq. 3:

$$
\begin{aligned}
A(t) & =\frac{\zeta^{2}\left(\gamma^{p}-h \gamma^{c}\right)}{4 O_{k}^{3} R_{g} T} \theta \\
R_{c}(t) & =\frac{\zeta^{2}\left(\gamma^{p}-h \gamma^{c}\right)}{4\left[\Delta G_{c}-U(h)-\Delta G_{U}-\Delta G_{\gamma}-\Delta G_{h}\right]}
\end{aligned}
$$

where $\zeta$ is a shape factor, and the terms $\Delta G_{U}, \Delta G_{\gamma}$, and $\Delta G_{h}$ describe the contributions to the critical particle radius 
Fig. 9 The temporal evolution of statistical information regarding the dispersions at a point in leg 1. Panels a and $\mathbf{c}$ present the evolution of the mean height and diameter of the cylindrical $\gamma^{\prime \prime}$ and $\delta$ precipitates, respectively. Panel figures $\mathbf{b}$ and d present the evolution of the precipitate volume fractions
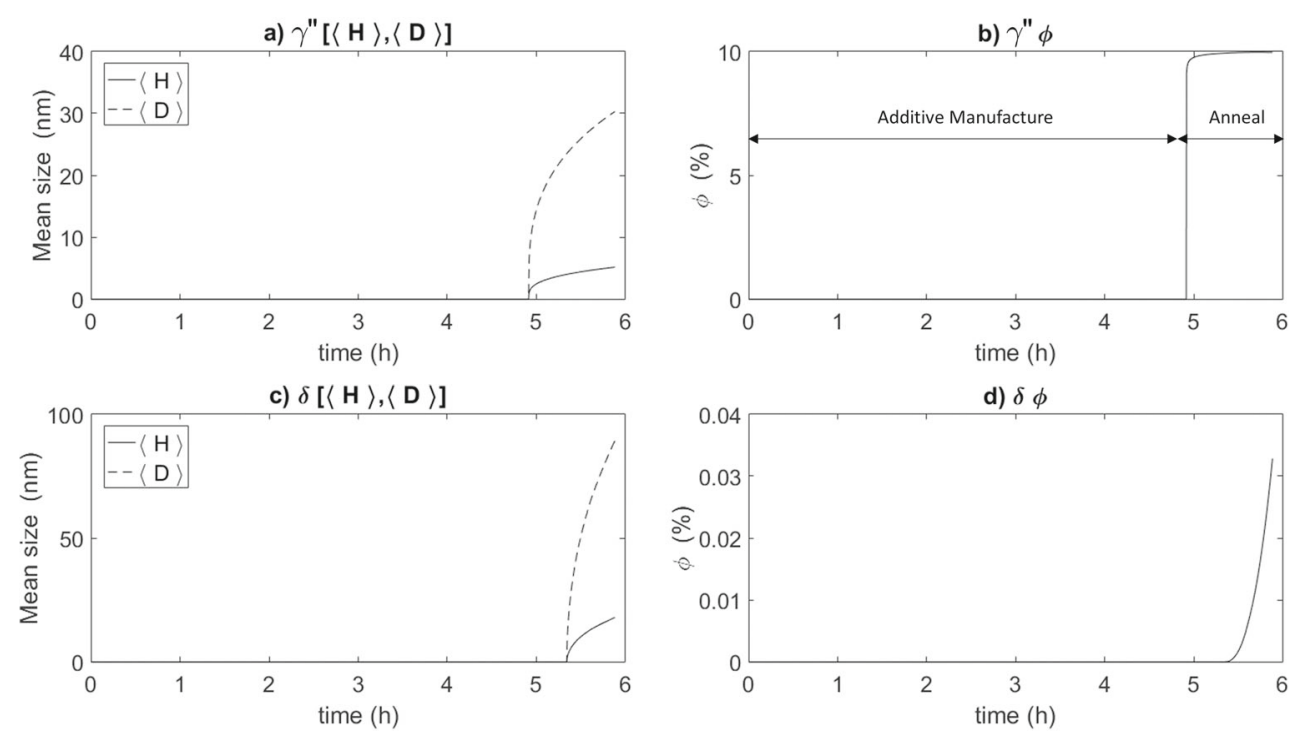

arising from changes to the misfit strain energy, interfacial energy, and aspect ratio, respectively. These terms are given below as follows:

$$
\begin{aligned}
\Delta G_{U} & =\frac{R}{3} \frac{d U(h)}{d R} \quad \Delta G_{\gamma}=\frac{\zeta^{2}}{8}\left(\frac{d \gamma^{p}}{d R}+\frac{d \gamma^{c}}{d R}\right) \\
\Delta G_{h} & =-\frac{\zeta^{2}}{12 h} \frac{d h}{d R}\left(\gamma^{p}-h \gamma^{c}\right)
\end{aligned}
$$

The impact of these factors upon precipitation kinetics can be discussed in terms of the classical regimes of precipitation: nucleation, growth, coarsening, and dissolution. For precipitates to nucleate, the critical particle radius must be smaller or equivalent to the critical nuclei radius. For a unimodal precipitate dispersion during the regime of growth, $R_{c}<\langle R\rangle$. During steady-state coarsening, $R_{c} \approx\langle R\rangle$, and during dissolution $R_{c}>\langle R\rangle$. Once the precipitate

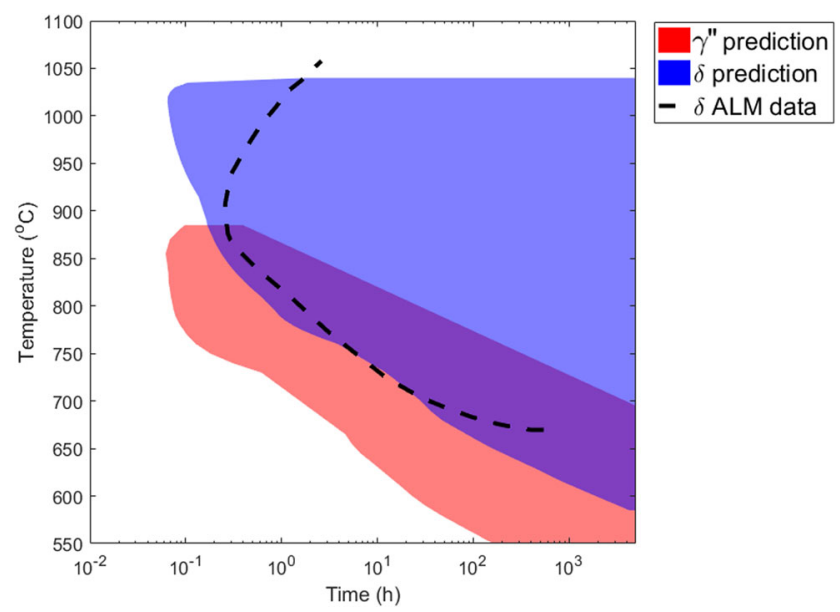

Fig. 10 The predicted time-temperature-transition behaviour within the Nb-rich region of a AM component. The data for Inconel 625 in the AM condition is measured by Stoudt et al. [21], and refers to the time needed for the formation of $\approx 1 \%$ volume fraction of $\delta$ reaches a dynamic equilibrium shape, misfit strain energy and interfacial energy contributions balance, and $\frac{d h}{d R} \rightarrow 0$.

The contributions to $R_{c}$ shown in Equation set (25) would diminish once the precipitates morphology changes sufficiently to reach a dynamic equilibrium shape, with reduced impact during coarsening kinetics compared to the regime of growth. The inclusion of these details may improve the ability to capture the rate at which the volume fraction of $\gamma^{\prime \prime}$ and $\delta$ are predicted to reach a dynamic equilibrium. The difficulties encountered when pursuing this approach is the determination of the planar and curved interfacial energies, and the misfit strains of the $\gamma^{\prime \prime}$ and $\delta$ phases. These vary as a function of composition and temperature. Atomistic calculations can provide an estimate for these parameters; however, such methods are difficult to extend to the high number of alloying elements commonly used nickel-based superalloys.

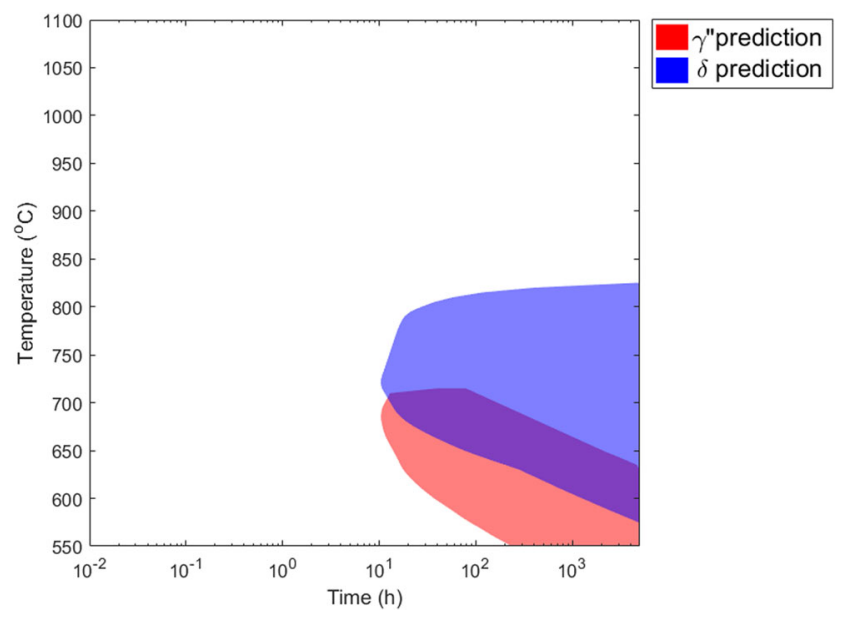

Fig. 11 The predicted time-temperature-transition behaviour within the $\mathrm{Nb}$-depleted region of a AM component 


\section{Conclusions}

- A mean-field theoretical framework has been developed for precipitation in nickel-based superalloys during $\mathrm{AM}$, which captures nucleation, growth, coarsening and dissolution.

- An extension to classical nucleation theory is presented that accounts for the influence of grain size and deformation upon precipitation.

- Evolution of the precipitate size distributions is handled through a multi-component, multi-phase description of particle growth rate as proposed by the SFFK model. The chemical driving force of stable and metastable phases can be calculated, capturing the competition between the $\gamma^{\prime \prime}$ and $\delta$ phases. The CALPHAD approach can link the impact of variations in chemistry upon precipitation behaviour.

- The precipitation model suggests that the anomalous precipitation behaviour exhibited by AM Inconel 625 during annealing is largely caused by the dendritic segregation of $\mathrm{Nb}$. No precipitation is predicted to form within the Nb-depleted regions. Differences in dislocation density and grain size are predicted to have a smaller impact upon precipitation behaviour in comparison to the role of dendritic segregation of $\mathrm{Nb}$.

- The low volume fraction of precipitates in Inconel 625 means that the mean-field assumption of weak interactions between precipitates is reasonable when modelling this alloy.

- To avoid the excessive precipitation of $\delta$, the anneal temperature needs to be high enough to homogenize $\mathrm{Nb}$ whilst minimising grain growth. If possible, $\mathrm{AM}$ process parameters and build strategies which minimise the segregation of $\mathrm{Nb}$ would help mitigate issues arising from the rapid precipitation kinetics of $\delta$ and $\gamma^{\prime \prime}$.

Acknowledgments The authors wish to thank colleagues who assisted in this project, including Richard Turner, Yogesh Sovani, David Gonzalez-Rodriquez, and Gavin Yearwood. Henrik Larsson provided great support and assistance in the application of ThermoCalc's TQ FORTRAN user interface. The computations described in this paper were performed using the University of Birmingham's BlueBEAR HPC service, which provides a High Performance Computing service to the University's research community. See http://www.birmingham. ac.uk/bear for more details.

Funding Information This work is funded by the Aerospace Technology Institute (ATI) through the Manufacturing Portfolio programme (project no. 113084).

\section{Compliance with Ethical Standards}

Conflict of Interest The authors declare that they have no conflict of interest.
Open Access This article is distributed under the terms of the Creative Commons Attribution 4.0 International License (http:// creativecommons.org/licenses/by/4.0/), which permits unrestricted use, distribution, and reproduction in any medium, provided you give appropriate credit to the original author(s) and the source, provide a link to the Creative Commons license, and indicate if changes were made.

\section{References}

1. Marchese G, Colera XG, Calignano F, Lorusso M, Biamino S, Minetola P, Manfredi D (2017) Characterization and comparison of Inconel 625 processed by selective laser melting and laser metal deposition. Adv Eng Mater 19(3):1-9

2. Lass EA, Stoudt MR, Williams ME, Katz M, Levine LE, Phan TQ, Gnaeupel-Herold TH, Ng DS (2017) Formation of the Ni3Nb delta-phase in stress-relieved Inconel 625 produced via laser powder-bed fusion additive manufacturing. Metall Mater Trans A 48A(11):5547-5558

3. Suave LM, Cormier J, Villechaise P, Soula A, Hervier Z, Bertheau D, Laigo J (2014) Microstructural evolutions during thermal aging of alloy 625: impact of temperature and forming process. Metall Mater Trans A 45(7):2963-2982

4. Amato K, Hernández J, Murr L, Martinez E, Gaytan SM, Shindo PW, Collins S (2012) Comparison of microstructures and properties for a Ni-base superalloy (Alloy 625) fabricated by electron and laser beam melting. Journal of Materials Science Research 1:3-41

5. Eiselstein HJD, Tillack JJ (1991) The invention and definition of alloy $6251-14$

6. Anderson MJ, Panwisawas C, Sovani Y, Turner RP, Brooks JW, Basoalto HC (2018) Mean-field modelling of the intermetallic precipitate phases during heat treatment and additive manufacture of Inconel 718. Acta mater 156:432-445

7. Panwisawas C, Qiu C, Anderson MJ, Sovani Y, Turner RP, Attallah MM, Brooks JW, Basoalto HC (2017) Mesoscale modelling of selective laser melting: thermal fluid dynamics and microstructural evolution. Comp Mater Sci 126:479-490

8. Panwisawas C, Sovani Y, Anderson MJ, Turner RP, Palumbo NM, Saunders BC, Choquet I, Brooks JW, Basoalto HC et al (2016) Multi-scale multi-physics approach to modelling of additive manufacturing in nickel-based superalloys. In: Hardy M (ed) Superalloys2016. Warrendale, TMS, pp 1021-1030

9. Capriccioli A, Frosi R (2009) Multipurpose ANSYS FE procedure for welding processes simulation. Fusion Eng Des 84(2-6):546553

10. Basoalto H, Anderson M (2016) An extension of mean-field coarsening theory to include particle coalescence using nearestneighbour functions. Acta Mater 117:112-134

11. Svoboda J, Fischer FD, Fratzl P, Kozeschnik E (2004) Modelling of kinetics in multi-component multi-phase systems with spherical precipitates: I: Theory. Mat Sci Eng A-Struct 385:166-174

12. Kozeschnik E, Svoboda J, Fischer FD (2006) Shape factors in modeling of precipitation. Mat Sci Eng A-Struct 441(1-2):68-72

13. Svoboda J, Fischer FD, Mayrhofer PH (2008) A model for evolution of shape changing precipitates in multicomponent systems. Acta Mater 56(17):4896-4904

14. Marqusee JA, Ross J (1984) Theory of Ostwald ripening competitive growth and its dependence on volume fraction. J Chem Phys 80:563-543

15. Jou HJ, Voorhees PW, Olson GB et al (2004) Computer simulations for the prediction of microstructure/property variation 
in aeroturbine disks. In: Green KA (ed) Superalloys 2004, pp 877886

16. Kocks UF, Mecking H (2003) Physics and phenomenology of strain hardening: the FCC case. Prog Mater Sci 48:171-273

17. Basoalto H (2010) Physical based continuum damage mechanics, personal communication

18. Andersson J, Helander T, Höglund L, Shi PF, Sundman B (2002) Thermo-Calc \& Dictra, computational tools for materials science. CALPHAD 26(2):273-312

19. Zhang F, Lyle LE, Andrew AJ, Stoudt MR, Lindwall G, Lass EA, Williams ME, Idell Y, Campbell CE (2018) Effect of heat treatment on the microstructural evolution of a nickel-based superalloy additive-manufactured by laser powder bed fusion. Acta Mater 152:200-214

20. Moore IJ, Burke MG, Palmiere EJ (2016) Modelling the nucleation, growth and coarsening kinetics of $\gamma^{\prime \prime}$ precipitates in the Ni-base alloy 625. Acta Mater 119:157-166
21. Stoudt MR, Lass EA, Ng DS, Williams ME, Zhang F, Campbell CE, Lindwall G, Levine LE (2018) The influence of annealing temperature and time on the formation of $\delta$-phase in additivelymanufactured Inconel 625. Metall and Mat Trans A 49A:30283037

22. Lindwall G, Campbell CE, Kass EA, Zhang F, Stoudt MR, Allen AJ, Levine LE (2019) Simulation of TTT curves for additively manufactured Inconel 625. Metall and Mat Trans A 50(1):457467

23. Brooks JW, Bridges PJ (1988) Metallurgical stability of Inconel alloy 718. Superalloys 1988:33-42

Publisher's Note Springer Nature remains neutral with regard to jurisdictional claims in published maps and institutional affiliations. 